\title{
Biosynthesis of angelyl-CoA in Saccharomyces cerevisiae
}

\author{
Roberta Callari ${ }^{1,2}$, David Fischer ${ }^{1}$, Harald Heider ${ }^{1}$ and Nora Weber ${ }^{1 *}$
}

\begin{abstract}
Background: The angelic acid moiety represents an essential modification in many biologically active products. These products are commonly known as angelates and several studies have demonstrated their therapeutic benefits, including anti-inflammatory and anti-cancer effects. However, their availability for use in the development of therapeutics is limited due to poor extraction yields. Chemical synthesis has been achieved but its complexity prevents application, therefore microbial production may offer a promising alternative. Here, we engineered the budding yeast Saccharomyces cerevisiae to produce angelyl-CoA, the CoA-activated form of angelic acid.

Results: For yeast-based production of angelyl-CoA we first expressed genes recently identified in the biosynthetic cluster ssf of Streptomyces sp. SF2575 in S. cerevisiae. Exogenous feeding of propionate and heterologous expression of a propionyl-CoA synthase from Streptomyces sp. were initially employed to increase the intracellular propionyl-CoA level, resulting in production of angelyl-CoA in the order of $5 \mathrm{mg} / \mathrm{L}$. Substituting the Streptomyces sp. propionyl-CoA carboxylase with a carboxylase derived from Streptomyces coelicolor resulted in angelyl-CoA levels up to $6.4 \mathrm{mg} / \mathrm{L}$. In vivo analysis allowed identification of important intermediates in the pathway, including methyl-malonyl-CoA and 3-hydroxyl-2-methyl-butyryl-CoA. Furthermore, methyl-malonate supplementation and expression of matB CoA ligase from S. coelicolor allowed for methyl-malonyl-CoA synthesis and supported, together with parts of the ssf pathway, angelyl-CoA titres of approximately $1.5 \mathrm{mg} / \mathrm{L}$. Finally, feeding of angelic acid to yeasts expressing acyl-CoA ligases from plant species led to angelyl-CoA production rates of approximately $40 \mathrm{mg} / \mathrm{L}$.
\end{abstract}

Conclusions: Our results demonstrate the biosynthesis of angelyl-CoA in yeast from exogenously supplied carboxylic acid precursors. This is the first report on the activity of the ssf genes. We envision that our approach will provide a platform for a more sustainable production of the pharmaceutically important compound class of angelates.

Keywords: Angelyl-CoA, Angelate, Propionyl-CoA, Methylmalonyl-CoA, 2-Methylacetoacetyl-CoA, 3-Hydroxyl-2methyl-butyryl-CoA, Metabolic engineering, CoA-ligase, Euphorbia peplus, Yeast

\section{Background}

Esters of angelic acid ((Z)-2-methyl-2-butenoic acid), also known as angelates, are pharmacologically active natural products widely distributed in plants (Additional file 1: Figure S1). The best-known and most studied example is certainly represented by ingenol-3-angelate (also known as ingenol-mebutate), a topical chemotherapeutic recently approved by the FDA for the treatment of actinic keratosis, a pre-cancerous skin condition. This ester of the diterpenoid ingenol and angelic acid is derived from

\footnotetext{
*Correspondence: noraw@evolva.com

${ }^{1}$ Evolva SA, Duggingerstrasse 23, 4153 Reinach, Switzerland

Full list of author information is available at the end of the article
}

the sap of Euphorbia peplus-the garden weed "petty spurge", known as a traditional remedy for warts and basal cell carcinomas [1]. Other notable plant angelates include petasin and its isomers isopetasin and neopetasin, found in Petasites hybridus, a species used for years as herbal supplement for its anti-spasmodic and anti-inflammatory effects [2]. The cytotoxic gordonosides, angelic acid esters isolated from Gordonia chrysandra, are frequently applied in traditional medicine for treating diarrhea, gastralgia, and arthritis [3]. Decoursinol angelate, isolated from roots of the medicinal herb Angelica gigas Nakai, exerts anti-cancer and anti-inflammatory activities [4]. Thapsigargin, found in the roots of the mediterranean plant species Thapsia garganica L., is an inhibitor of the 
sarco-endoplasmic reticulum $\mathrm{Ca}^{2+}$-ATPase used in the treatment of solid tumors [5].

Recently, bacterial angelates have also been reported: SF2575 is a tetracycline polyketide-angelic acid ester produced by Streptomyces sp. SF2575 [6]. The compound exhibits not only weak antibiotic activity but also potent anti-cancer activity towards a broad range of cancer cell lines [7]. Trehangelins are trehalose angelates produced by the endophytic actinomycete Polymorphospora rubra K07-0510, displaying potent inhibitory activity against hemolysis of red blood cells [8].

Supply of angelates is currently based on extraction of the pure compounds from the species of origin. In some cases chemical synthesis has also been achieved [9-12]. However, both approaches are low yielding and have high environmental impact. In the case of ingenol-mebutate, direct isolation from the aerial tissue of E. peplus only yields $1.1 \mathrm{mg} / \mathrm{kg}$ of tissue [13]. Semi-synthesis, starting from the more abundant cognate compound ingenol, obtained an overall yield of around $31 \%$, but relied on expensive catalysts [10]. Thapsigargin is isolated from wild plants of $T$. garganica, where it is present in minute amounts $(1.2-1.5 \%$ of the dry weight, depending on the selected tissue) [5]. Its total synthesis in 42 steps from (S)-carvone had an overall yield of only $0.6 \%$ [9].

To make these compounds more accessible, microbial production certainly represents an interesting alternative route.

Unfortunately, biosynthesis of any of the plant angelates has not yet been elucidated and even the enzymes involved in the biosynthesis of the angelic acid moiety are unknown. In contrast, the gene clusters responsible for the bacterial synthesis of SF2575 ("ssf") and trehangelin A ("thg") have been identified and characterized $[6,14]$. This has led to the elucidation of the metabolic pathways responsible for the biosynthesis of these compounds and the identification of enzymes needed for assembly of their core structures and also for tailoring reactions, including angelyl-CoA (AN-CoA) formation and esterification. The latter is synthesized in both Streptomyces sp. SF2575 and P. rubra by enzymes resembling those found in fatty acid biosynthesis. In joint action, the identified beta-ketoacyl-(acyl-carrier-protein) synthase III (KAS III), the 3-ketoacyl-(acyl-carrier-protein) reductase and the enoyl-CoA hydratase may lead to AN-CoA biosynthesis starting from acetyl-CoA (Ac-CoA) and methyl-malonyl-CoA (MM-CoA) via the intermediates 2-methyl-acetoacetyl-CoA (MAA-CoA) and 3-hydroxyl2-methyl-butyryl-CoA (HMB-CoA) (Fig. 1). The enzymes from $P$. rubra were characterized in vitro [14], whereas the enzymes from S. sp. SF2575 have been suggested to be involved in AN-CoA formation based on homology to functionally similar enzymes from other species [6]. Pickens and co-workers [6] proposed that in S. sp. SF2575 condensation of MM-CoA and Ac-CoA to MMA-CoA is catalyzed by SsfN, a KAS III homolog. SsfK, homologous to 3-oxoacyl-ACP reductases, enables keto-reduction of MAA-CoA yielding HMB-CoA. In the last step, stereospecific dehydration of HMB-CoA by Ssf) (a member of the enoyl-CoA hydratase/isomerase family) results in AN-CoA. Moreover, SsfE, homologous to biotin-dependent methyl-malonyl-CoA decarboxylases and propionyl-CoA carboxylases, was suggested to be involved in MM-CoA formation from Pr-CoA.

In this study we describe production of angelyl-CoA in the yeast Saccharomyces cerevisiae. This production host has well-established advantages when compared to other microorganisms such as robustness and resistance under harsh industrial conditions, resistance to phages and the ability to ferment sugars under acidic conditions. AN-CoA biosynthesis was achieved through expression of a heterologous pathway derived from the bacterial ssf cluster, allowing $\mathrm{AN}-\mathrm{CoA}$ synthesis starting from propionyl-CoA. Upon engineering of the propionyl-CoA metabolism we reached maximum titres of approximately $6.4 \mathrm{mg} / \mathrm{L}$ AN-CoA. Moreover, the identification of

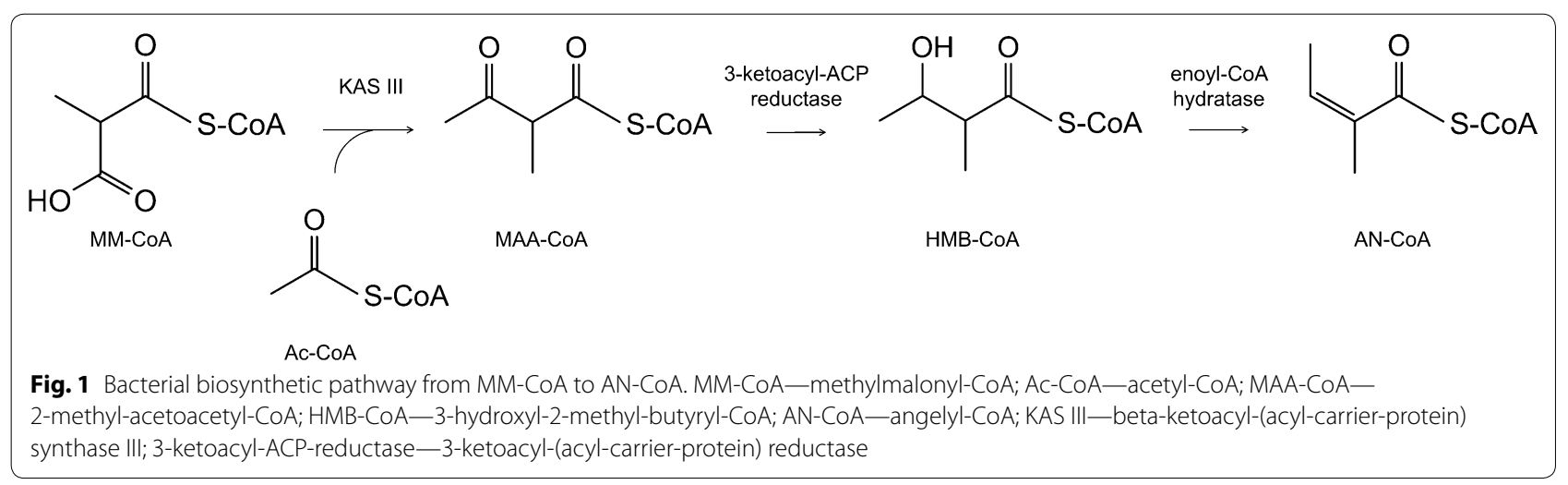


acyl-CoA synthases from different plant sources enabled the use of angelic acid as substrate, yielding AN-CoA in titres of approximately $40 \mathrm{mg} / \mathrm{L}$.

AN-CoA represents an important building block for the synthesis of many plant secondary metabolites with biological activity. AN-CoA is the substrate used by acylCoA transferases to catalyse esterification reactions, adding the angelate moiety onto diverse acceptor molecules. Its biosynthesis in yeast will enable angelyl-acylation of a broad range of compounds. Although further optimization is needed, we anticipate that the strains reported here will pave the way for the bio-based production of esters of angelic acid.

\section{Results}

Angelyl-CoA production in yeast starting from propionate Based on the hypotheses of Pickens et al. [6] we assembled the pathway to AN-CoA biosynthesis in baker's yeast. First, we expressed the bacterial genes $s s f E$, $s s f N$, $s s f K$, and $s s f J$ as yeast codon-optimized versions for the conversion of Pr-CoA into AN-CoA (Fig. 2a). Pr-CoA is an intermediate metabolite produced in yeast through a variety of pathways including thio-esterification of propionate and catabolism of odd chain fatty acids and selected amino acids [15]. A plasmid, co-expressing the four heterologous genes under control of strong constitutive promoters was constructed together with a control plasmid (no ORFs downstream of the promoters). Transformation into yeast generated strains ANG1 (control) and ANG2 (ssfE/ssfN/ssfK/ssf)). These strains were tested for production of AN-CoA. In strain ANG2 around $0.37 \mathrm{mg} / \mathrm{L}$ of AN-CoA accumulated, whereas no ANCoA could be detected in strain ANG1 (control strain, data not shown).

To test whether an improved precursor supply may increase $\mathrm{AN}-\mathrm{CoA}$ synthesis, we cultured the strains in propionic acid-supplemented medium. Propionic acid is metabolized by the yeast via activation to Pr-CoA, which is catalyzed by ACS1-an endogenous isoenzyme of acetyl-CoA synthase [16]. ACS1 can accept propionic acid as substrate, albeit at lower rates than acetic acid [17]. To further improve propionate thio-esterification, we then expressed an acyl-CoA synthase specific for this anion. We chose to express propionyl-CoA synthase prpE from Salmonella enterica serovar Typhimurium, encoding an enzyme required for the catabolism of propionate in this bacterium [18]. Two strains were generated, one expressing the ssf genes and an empty plasmid (ANG3), the other one expressing the ssf genes together with prpE under control of the PGK1 promoter (ANG4). Feeding with propionic acid led to 5.5-fold elevated intracellular accumulation of $\mathrm{Pr}-\mathrm{CoA}$ in strain ANG3, compared to the non-fed strain (Fig. 2b). Upon propionate supplementation, prpE expression in strain ANG4 boosted Pr-CoA accumulation to 20-fold higher values than those seen with strain ANG3 not expressing $\operatorname{prpE}$ (Fig. 2b). This is in accordance with an earlier report on $\operatorname{prp} E$ expression in the presence of exogenous propionate, leading to substantial accumulation of Pr$\mathrm{CoA}$ in yeast [19]. The elevated concentration of $\mathrm{Pr}-\mathrm{CoA}$ inside the cells resulted in a massive increase in AN-CoA production (from $0.37 \mathrm{mg} / \mathrm{L}$ to almost $5 \mathrm{mg} / \mathrm{L}$ at $12 \mathrm{~h}$ of growth, see Fig. 3a) but it also affected cell growth. The increased energy requirements needed to maintain cytosolic $\mathrm{pH}$ homeostasis in the presence of propionic acid in the medium may, in addition, contribute to growth retardation [20]. We cultured the cells in medium buffered to $\mathrm{pH} 4.5$ as this $\mathrm{pH}$ value is a compromise between support of a decent yeast growth and remaining below the $\mathrm{pK}_{\mathrm{a}}$ of propionic acid $\left(\mathrm{pK}_{\mathrm{a}}=4.88\right)$. This $\mathrm{pH}$ supports passive diffusion of propionic acid into the cells [21]. Cells cultured at $\mathrm{pH} 4.5$ showed better growth and a slightly elevated production of AN-CoA (Fig. 2c).

We then performed time course experiments with strain ANG4 (prpE + ssfE/ssfN/ssfK/ssff; Fig. 3a) and a newly constructed strain, ANG5 ( $p r p E+p c c B / a c c A 1 /$ $s s f N / s s f K / s s f$; Fig. $3 \mathrm{~b}$ ). The latter strain expresses the propionyl-CoA carboxylase complex from Streptomyces coelicolor [22], instead of ssfE. The complex consists of the transcarboxylase subunit PccB and the biotin carrier protein/biotin carboxylase subunit AccA1 (PccB/ AccA1 complex). Both strains were grown in buffered, propionate-supplemented medium. AN-CoA formation peaked at $12 \mathrm{~h}$, but could barely be detected after $48 \mathrm{~h}$ of culture. AN-CoA accumulation reached maximally $4.9 \pm 0.5 \mathrm{mg} / \mathrm{L}$ in strain ANG4 (Fig. 3a), whereas it climbed to even $6.4 \pm 0.2 \mathrm{mg} / \mathrm{L}$ in strain ANG5 after $12 \mathrm{~h}$ of growth (Fig. 3b). Similar dynamics were detected for Ac-CoA, a critical metabolite for growth and proliferation. Ac-CoA accumulation also peaked at $12 \mathrm{~h}$ and went down to almost 0 upon $96 \mathrm{~h}$ of shake flask culture. In order to correlate AN-CoA production with $\mathrm{Pr}-\mathrm{CoA}$ production, we also analyzed the relative amount of $\mathrm{Pr}-\mathrm{CoA}$ accumulation in strains ANG4 and ANG5. Both strains accumulated similar levels of $\mathrm{Pr}-\mathrm{CoA}$ within the first $12 \mathrm{~h}$. Thereafter, in strains expressing $p c c B / a c c A 1$, Pr$\mathrm{CoA}$ remained stable up to at least $36 \mathrm{~h}$ of growth. Even after $96 \mathrm{~h}$ a significantly higher level of $\mathrm{Pr}-\mathrm{CoA}$ could be detected in ANG5 expressing $p c c B / a c c A 1$ than in ANG4 expressing $s s f E$ (Fig. 3c).

\section{Further analyses of the angelyl-CoA pathway in yeast}

In order to investigate the individual functions of the $s s f$ genes involved in AN-CoA biosynthesis in vivo, three truncated pathways were assembled on plasmids and transformed into yeast (strains ANG6-ANG8). The three 

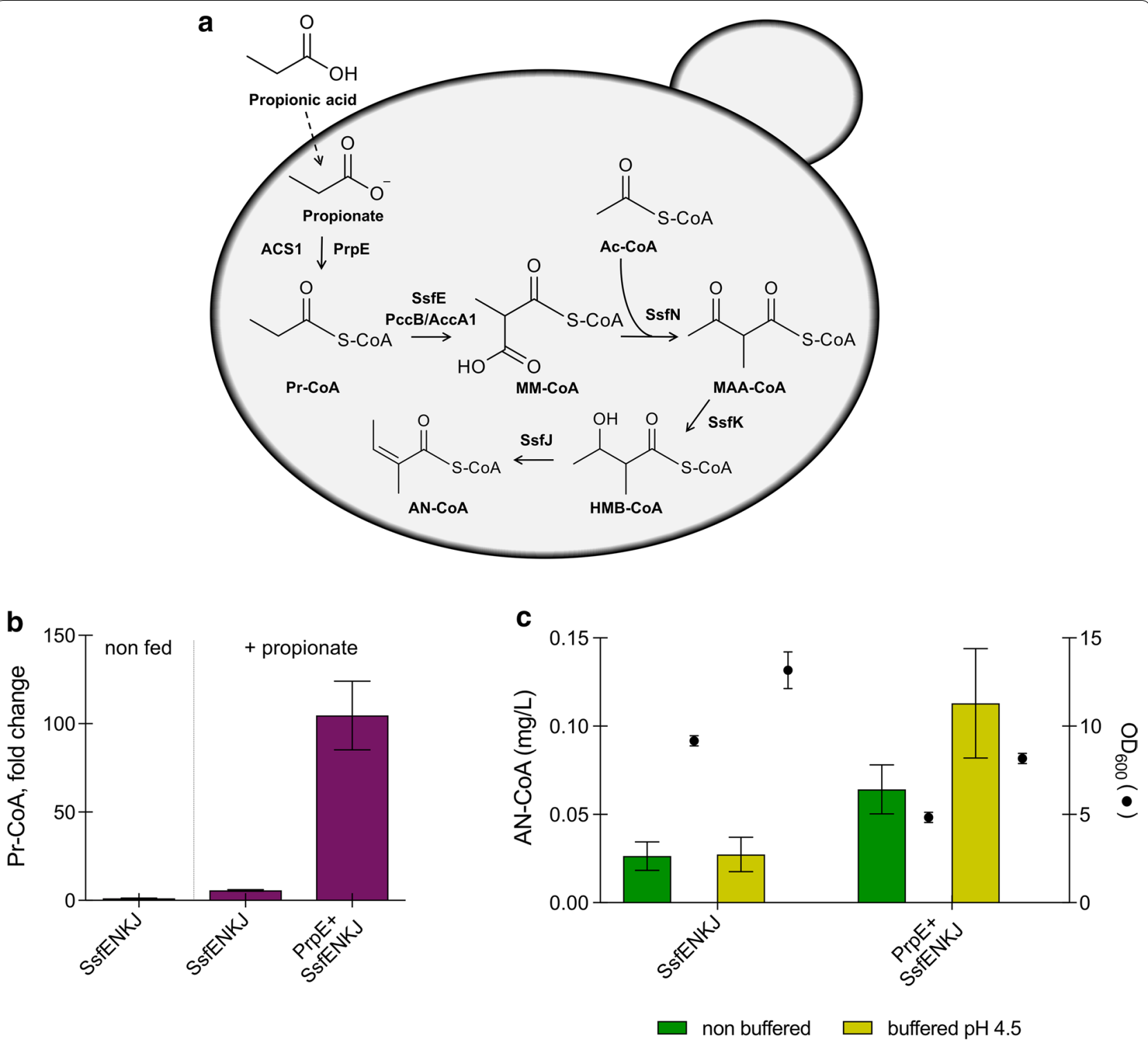

Fig. 2 Intracellular accumulation of Pr-CoA and AN-COA in S. cerevisiae strains expressing the SSFENKJ genes. a Graphical representation of the pathway starting with propionic acid feeding. b Pr-COA accumulation in strains expressing ssfENKJ, and ssfENKJ together with prpE, shown as fold change compared to the respective control strains. Engineered strains were incubated in selective SC medium either non supplemented ("non fed") or supplemented with $0.5 \mathrm{~g} / \mathrm{L}$ propionic acid ("+ propionate"). c Intracellular accumulation of AN-CoA in strains ANG3 (ssfENKJ) and in strain ANG4 (prpE + ssfENKJ) grown for $72 \mathrm{~h}$ in SC medium supplemented with $0.5 \mathrm{~g} / \mathrm{L}$ propionic acid. The medium was buffered to $\mathrm{pH} 4.5$ (yellow bars) or supplied unbuffered (green bars). Circles indicate $\mathrm{OD}_{600}$ at $72 \mathrm{~h}$ of growth. Represented are the averages and standard deviations of three independent cultures

strains expressed prpE together with one (ssfE, ANG6), two (ssfE/ssfN, ANG7), or three (ssfE/ssfN/ssfK, ANG8) genes from the $s s f$ pathway. These strains were analysed together with the control strain expressing solely prpE (ANG9), and the strain expressing the entire pathway (prpE $+s s f E / s s f N / s s f K / s s f j$, ANG4). Strains were analysed for production of $\mathrm{Pr}-\mathrm{CoA}, \mathrm{AN}-\mathrm{CoA}$ and the putative intermediates MM-CoA, MAA-CoA and HMB-CoA (see
Additional file 1: Figure S2 for extracted ion chromatograms of all strains).

The strain expressing solely prpE (ANG9) accumulated only Pr-CoA (Fig. 4). The same is true for ANG6 ( $p r p E$ and $s s f E$ expressed). Neither MM-CoA nor MAA-CoA could be detected in strains expressing $p r p E / s s f E / s s f N$ (ANG7). However, accumulation of $\mathrm{Pr}-\mathrm{CoA}$ in those strains was much lower compared to control strain 

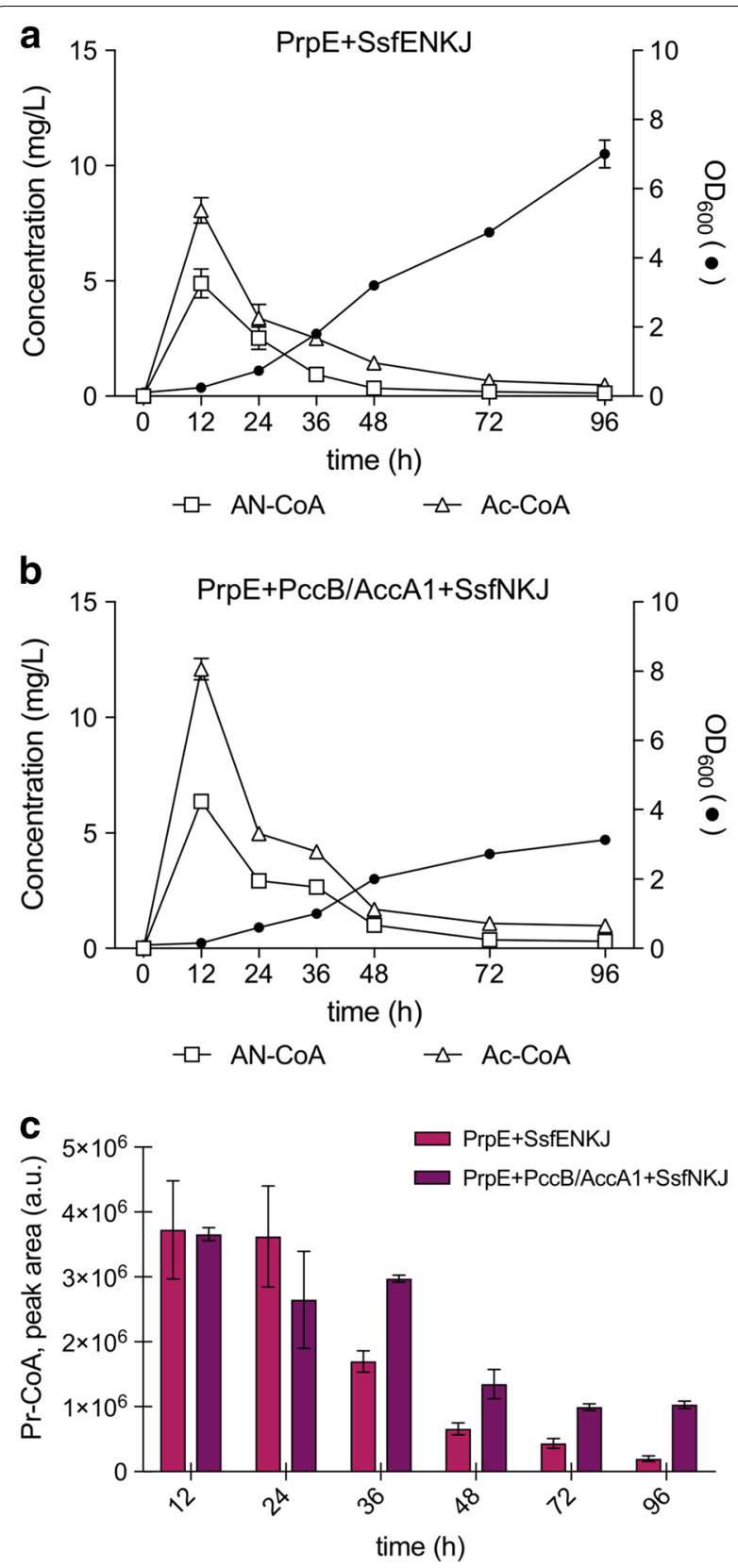

Fig. 3 Time course of production of AN-COA, Ac-CoA and Pr-CoA. a $A c-C O A$ and $A N-C O A$ produced by strain expressing prpE + ssfENKJ (ANG4). $\mathbf{b}$ Ac-CoA and AN-CoA produced by strain expressing $p r p E+p c c B / a c c A 1+s s f N K J$ (ANG5). $\mathbf{c}$ Relative amounts of Pr-CoA in strains expressing prpE + ssfENKJ (ANG4) and strains expressing $p r p E+p c c B / a c c A 1+s S f N K J$ (ANG5). Strains were grown in SC medium supplemented with $0.5 \mathrm{~g} / \mathrm{L}$ propionic acid and buffered to $\mathrm{pH} 4.5$. Filled circles in $\mathbf{a}, \mathbf{b}$ indicate $\mathrm{OD}_{600}$. Represented are the averages and standard deviations of three independent cultures

ANG9, suggesting that $\operatorname{Pr}$-CoA might have been utilized for further reactions inside the cells. When the pathway was extended to include $s s f K$ (ANG8) yeasts produced HMB-CoA. In addition, a substantial accumulation of
MM-CoA $(1.7 \mathrm{mg} / \mathrm{L})$ was detected in this strain, together with higher levels of Pr-CoA, compared to strains ANG6 or ANG7 (nearly seven- and ninefold more, respectively). Finally, strain ANG4, containing the entire pathway, accumulated AN-CoA together with lower amounts of HMB-CoA (nearly twofold less than ANG8) and MM-CoA (7.5-fold less than ANG11). Pr-CoA levels were comparable to those found in the control strain expressing only prpE (Fig. 4). Over time accumulation of HMB-CoA in strains expressing $\mathrm{prpE} / \mathrm{ssfE} / \mathrm{ssfN} / \mathrm{ssfK}$ and accumulation of AN-CoA in strains expressing prpE/ ssfE/ssfN/ssfK/ssfJ was accompanied by a decrease of the earlier intermediates (Additional file 1: Figure S3).

\section{Angelyl-CoA production in yeast starting from methyl-malonate}

As an alternative route to $\mathrm{AN}-\mathrm{CoA}$ production, we evaluated the malonyl/methylmalonyl-CoA ligase operating naturally in Streptomyces coelicolor. The biosynthetic route should allow for MM-CoA synthesis in yeast upon methyl-malonate supplementation and heterologous expression of malonyl-CoA synthase matB (Fig. 5a). MatB is an enzyme exhibiting a certain promiscuity, accepting both malonate and methyl-malonate as substrates [23]. Building of this pathway may avoid accumulation of potentially toxic amounts of $\mathrm{Pr}-\mathrm{CoA}$ as it starts with a different substrate for production of methyl-malonyl-CoA.

Three plasmids were constructed and transformed into yeast, thus generating strain ANG10 (expressing matB), strain ANG11 (expressing ssfN/ssfK/ssf) and strain ANG12 (expressing the entire pathway matB $+s s f N / s s f K /$ $s s f)$ ). Without feeding of methyl-malonic acid MM-CoA could not be detected in those strains (data not shown). Upon methyl-malonate feeding, expression of matB in strain ANG10 led to around $2.7 \mathrm{mg} / \mathrm{L} \mathrm{MM-CoA} \mathrm{accu-}$ mulation, representing the majority of the acyl CoA-pool (Fig. 5b). As expected, none of the compounds of interest could be detected in strain ANG7 expressing exclusively the $s s f N / s s f K / s s f J$ genes. Strain ANG12, expressing the entire pathway $(m a t B+s s f N / s s f K / s s f)$, was able to produce not only MM-CoA but also AN-CoA in titres in the range of $1.3-1.9 \mathrm{mg} / \mathrm{L}$ (Fig. $5 \mathrm{~b}, \mathrm{c}$ ). Interestingly, also Pr-CoA substantially accumulated in strain ANG12 (matB $+s s f N / s s f K / s s f)$ (Fig. 5b). This was not observed in strain ANG10 or ANG11, suggesting that the accumulation was induced only when the full pathway was expressed. As shown with propionic acid feeding of strains ANG4 and ANG5 (Fig. 3a, b), AN-CoA accumulation peaked at $12 \mathrm{~h}$ of growth and declined thereafter to levels close to the limit of detection (Fig. 5c). MM-CoA accumulated in this strain to maximal titres of $6.1 \pm 0.1 \mathrm{mg} / \mathrm{L}$ at $12 \mathrm{~h}$. 


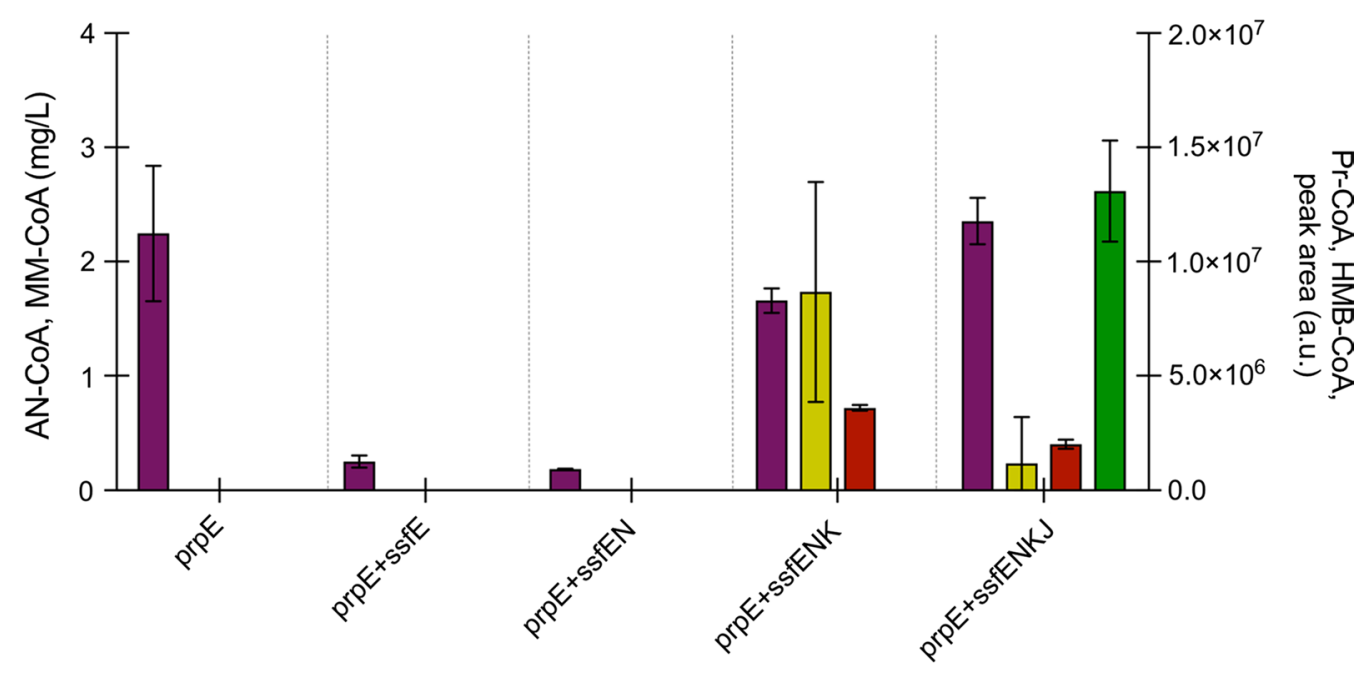

\section{Pr-CoA $\square$ MM-CoA $\square$ HMB-CoA $\square$ AN-CoA}

Fig. 4 Intracellular accumulation of $\operatorname{Pr}-\mathrm{COA}, \mathrm{MM}-\mathrm{COA}, \mathrm{HMB}-\mathrm{COA}, \mathrm{AN}-\mathrm{COA}$ in strains expressing parts of the AN-CoA pathway. MAA-CoA could not be found in any of the strains. Pr-CoA (purple bars), MM-COA (yellow bars), HMB-CoA (red bars) and AN-CoA (green bars) in strains expressing $p r p E$ (ANG9), prpE + ssfE (ANG6), prpE + ssfE/ssfN (ANG7), prpE + ssfE/ssfN/ssfK (ANG8) and prpE + ssfE/ssfN/ssfK/ssfJ (ANG4). Engineered strains were incubated for $12 \mathrm{~h}$ in selective SC medium buffered to $\mathrm{pH} 4.5$ and supplemented with $0.5 \mathrm{~g} / \mathrm{L}$ propionic acid. Represented are the averages and standard deviations of three independent cultures

\section{Angelic acid feeding and angelyl-CoA production}

In addition to setting up the entire pathway for angelyl-CoA production in yeast, we attempted to produce AN-CoA directly from angelic acid. We grew yeast strains expressing heterologous acyl-CoA ligases from plant, bacterial and fungal origin in angelic acid-supplemented medium (schematically shown in Fig. 6a). AcylCoA ligases can catalyze acyl-CoA thioester formation through adenylation of the carboxylic acid substrate. Many studies have explored the substrate specificity of these enzymes, revealing in several cases remarkable substrate promiscuities beyond their canonical substrate pools [24-26].

In preliminary experiments we found that two of the CoA-ligases tested showed the ability to accept angelic acid as a substrate for thio-esterification: carboxyl CoA ligase 4 from Humulus lupulus (HICCL4) and predicted acyl-activating enzyme 6 from Solanum tuberosum (StCCL). The sequences of those two CoA ligases were used to search for CoA ligases in the available transcriptome of Euphorbia peplus, the plant producing ingenol-3-angelate. We identified several potential candidates. Three of them, arbitrarily named EpCCL1, EpCCL2 and EpCCL3, were prioritized based on their high sequence identity to HICCL4 and StCCL (Additional file 1: Figure S4).

Three individual strains were constructed expressing the codon-optimized variants of EpCCL1 (ANG16), EpCCL2 (ANG17) or EpCCL3 (ANG18). Strains ANG16, ANG17, and ANG18, in parallel with strains expressing HlCCL4 (ANG14) and StCCL (ANG15), were assayed for AN-CoA production using medium supplemented with $0.1 \mathrm{~g} / \mathrm{L}$ angelic acid. Figure $6 \mathrm{~b}$ shows the relative activity of the CCL enzymes in yeast, expressed as percentage of AN-CoA accumulating in those cells. All of the putative CoA-ligases showed activity against angelic acid. Yeasts expressing the CoA ligase from S. tuberosum (ANG15) accumulated the highest amount of AN-CoA after $24 \mathrm{~h}$. The amount of AN-CoA accumulating in those cells was set to $100 \%$. ANG14, the strain expressing HlCCL4, accumulated $75 \%$ of this amount of AN-CoA, whereas yeasts expressing EpCCL1 (ANG16) or EpCCL2 (ANG17)

(See figure on next page.)

Fig. 5 Impact of methyl-malonic acid feeding on AN-CoA production. a Graphical representation of the pathway starting with methyl-malonic acid feeding. b Intracellular accumulation of Ac-CoA (orange bars), Pr-CoA (purple bars), MM-CoA (yellow bars), HMB-CoA (red bars) and AN-CoA (green bars) in strains expressing matB (ANG10), ssfNKJ (ANG11) and matB + ssfNKJ (ANG12), upon 12 h of growth. c Time course of production of AN-CoA (open squares), MM-CoA (open circles) and Ac-CoA (filled triangles) in strain expressing matB + ssfNKJ (ANG12), and corresponding optical density at $600 \mathrm{~nm}$ (filled circles). All engineered strains were incubated in selective SC medium, supplemented with $0.5 \mathrm{~g} / \mathrm{L}$ methyl-malonic acid (all data: mean $\pm S D, n=3)$ 

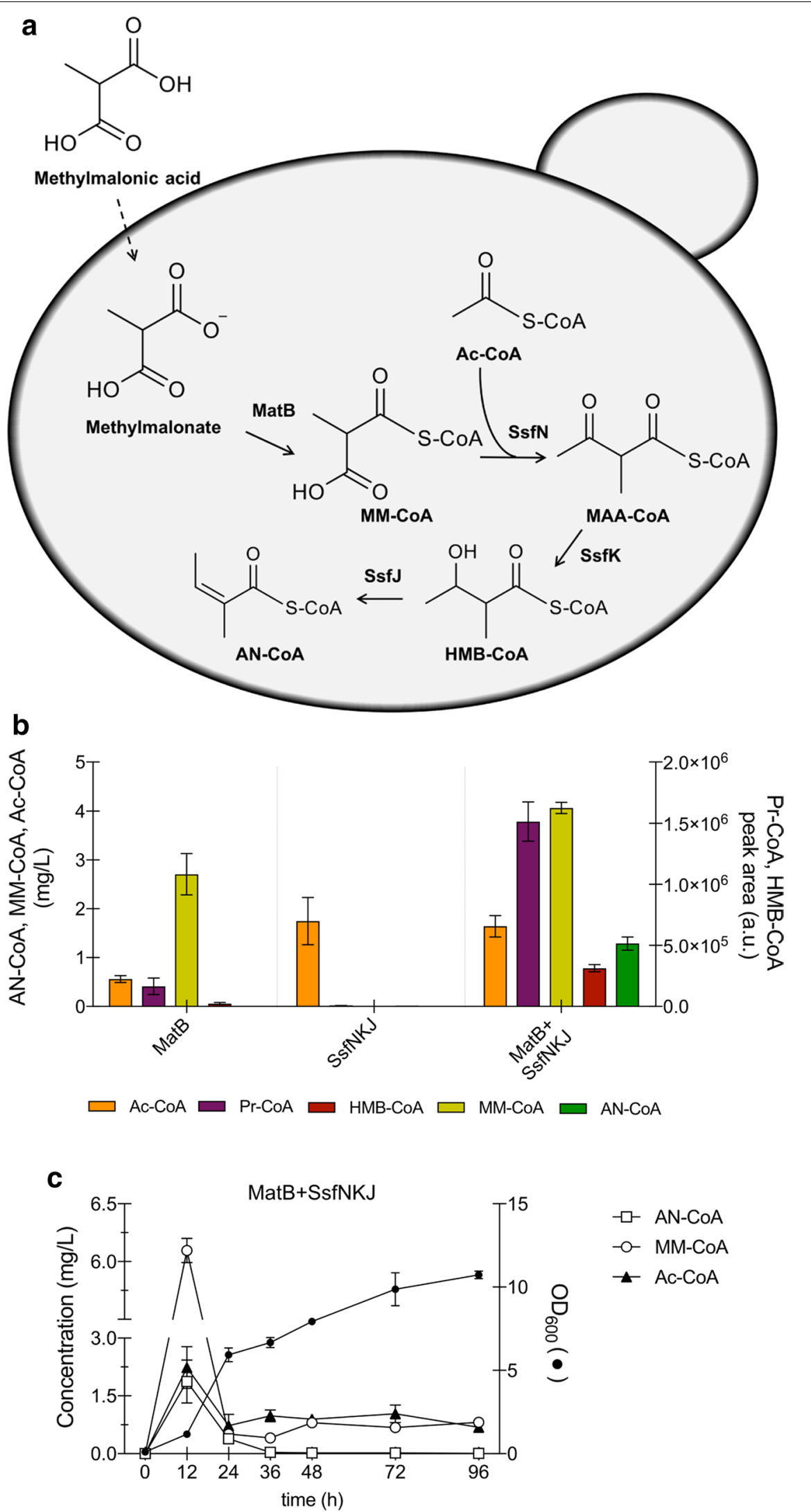


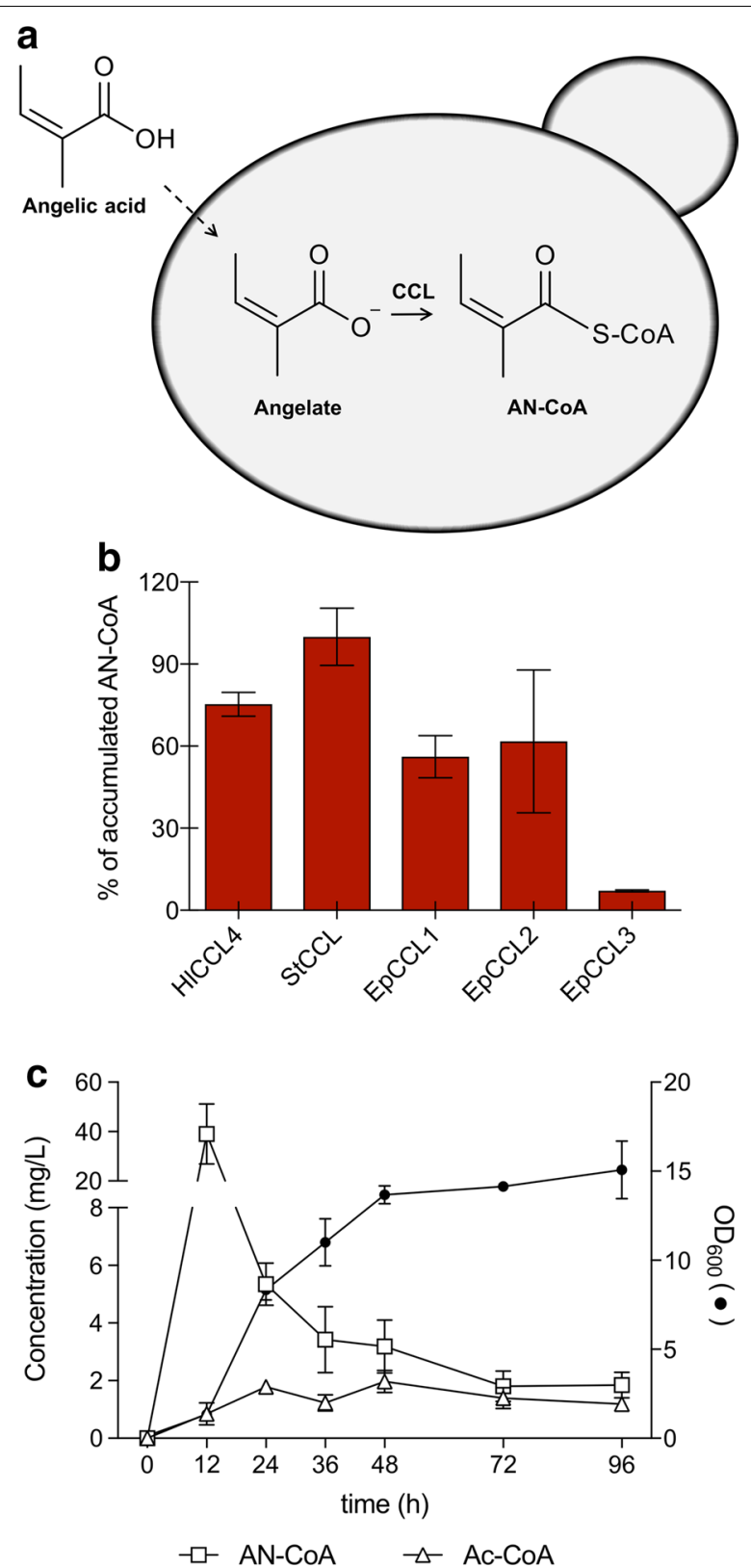

Fig. 6 AN-CoA production in strains expressing plant acyl-CoA ligases and fed with angelic acid. a Angelate enters the cell and is converted to angelyl-CoA by carboxyl CoA ligases. b Relative amount of AN-CoA accumulating in yeast strains expressing CoA-ligases HICCL4 (ANG14), StCCL (ANG15), EpCCL1 (ANG16), EpCCL2 (ANG17), and EpCCL3 (ANG18). Strains were cultured in selective SC medium supplemented with $0.1 \mathrm{~g} / \mathrm{L}$ angelic acid. c Time course of production of AN-CoA (open squares) and Ac-CoA (open triangles) by the strain expressing CoA-ligase StCCL (ANG15). The corresponding $\mathrm{OD}_{600}$ values are represented by filled circles. Engineered strains were incubated in selective SC medium supplemented with $0.1 \mathrm{~g} / \mathrm{L}$ angelic acid (all data: mean $\pm S D, n=3$ ) accumulated 56 or $62 \%$ of this amount of AN-CoA, respectively. The strain expressing EpCCL3 accumulated marginal amounts of $\mathrm{AN}-\mathrm{CoA}$ ( $7 \%$ of the amount of ANG15). Expression of the heterologous enzymes had negligible effect on strain growth (data not shown).

Time course analysis of the StCCL expressing strain revealed a peak in AN-CoA production after $12 \mathrm{~h}$, followed by a net decrease in titre (Fig. 6c). These kinetics were similar to the ones described before for AN-CoA production in yeast expressing the bacterial ssf pathway. The highest production level of AN-CoA at $12 \mathrm{~h}$ of batch culture was $39.0 \pm 9.9 \mathrm{mg} / \mathrm{L}$.

\section{Discussion}

In this study we demonstrated the potential of yeast to produce AN-CoA by (A) heterologous expression of genes from a recently discovered gene cluster derived from actinomycetes, (B) the heterologous expression of plant acyl-CoA synthases able to accept angelic acid as substrate.

\section{Expression of the ssf pathway in S. cerevisiae}

First, we showed production of AN-CoA in yeast through expression of part of the $s s f$ pathway adopted from Streptomyces sp. SF2575. Pr-CoA is converted by SsfE to MM-CoA, which serves as substrate for the subsequent condensation reaction with Ac-CoA. Pr-CoA and AcCoA are both present in yeast, albeit the former only in small amounts. Upon propionate supplementation, ten times more AN-CoA (almost $5 \mathrm{mg} / \mathrm{L}$ ) was produced in buffered medium when expressing an additional heterologous CoA-ligase, specific for Pr-CoA (propionyl-CoA synthase from Salmonella, PrpE).

Interestingly, the intracellular Pr-CoA concentration reached high levels, even when a second copy of the $s f f E$ gene was expressed (Additional file 1: Figure S5). This doubling of the gene dosage should optimize coupling of Pr-CoA synthesis and the further downstream steps, pulling most of the Pr-CoA to AN-CoA. We propose that Pot1, the only 3-ketoacyl-CoA thiolase present in S. cerevisiae, may be responsible for $\mathrm{Pr}-\mathrm{CoA}$ accumulation through the breakdown of the AN-CoA pathway intermediate MAA-CoA. Pot1 is normally involved in $\beta$-oxidation and it catalyses the conversion of 3-ketoacylCoA into an acyl-CoA shortened by two carbon atoms [27]. Shortening of MAA-CoA by two carbon atoms may inevitably lead to generation of $\mathrm{Pr}-\mathrm{CoA}$ and $\mathrm{Ac}-\mathrm{CoA}$, thus explaining $\mathrm{Pr}-\mathrm{CoA}$ build-up. The accumulation of Pr-CoA, besides being responsible for loss of carbon flux towards AN-CoA, may also be responsible for the growth inhibition suffered by those strains. Strains expressing ssfE reached $\mathrm{OD}_{600}$ values of $\sim 7$ after $96 \mathrm{~h}$ of growth, whereas strains expressing the $p c c B / a c c A 1$ complex, 
instead of $s s f E$, underwent a more severe growth inhibition $\left(\mathrm{OD}_{600}=3\right.$ after $\left.96 \mathrm{~h}\right)$, parallel to the elevated accumulation of Pr-CoA (Fig. 3a, b). It has been described for both the filamentous fungus Aspergillus nidulans and the bacterium Rhodobacter sphaeroides that Pr-CoA inhibits enzymes involved in glucose metabolism, in particular CoA-dependent enzymes such as pyruvate dehydrogenase and succinyl-CoA synthase, leading to a significant growth retardation [28, 29]. In Escherichia coli, Pr-CoA was found to be a competitive inhibitor of citrate synthase [30]. Similar mechanisms could explain the severe growth retardation observed in yeast upon accumulation of Pr-CoA.

We attempted to overcome Pr-CoA toxicity by employing an alternative biosynthetic route that directly produced MM-CoA upon expression of methyl-malonylCoA synthase matB from Streptomyces coelicolor. This enzyme has recently been shown to generate methylmalonyl-CoA in yeast upon methyl-malonate feeding [19]. Combining mat $B$ with the $s s f$ pathway genes indeed induced generation of AN-CoA without severely hampering cell growth. Cells grown in those conditions could reach an $\mathrm{OD}_{600}$ of 10.7 after $96 \mathrm{~h}$ of growth, compared to $\mathrm{OD}_{600}$ of 3 and 7 when supplementing propionic acid (see Figs. 3, 5). Nevertheless, the levels of AN-CoA $(1.5 \mathrm{mg} / \mathrm{L})$ did not reach the values seen in yeast operating with the Pr-CoA pathway. The fact that expression of this alternative pathway also induces accumulation of $\mathrm{Pr}-\mathrm{CoA}$ is consistent with the previously formulated hypothesis that $\mathrm{Pr}-\mathrm{CoA}$ accumulation is induced via Pot1 activity on MAA-CoA.

The individual steps of the pathway were further characterized in vivo by sequential expression of the corresponding pathway genes. Contrary to expectations, no MM-CoA nor MAA-CoA could be detected in strains expressing either only $s s f E$, or $s s f E$ together with $s s f N$. In cells expressing the entire pathway, accumulation of MMCoA was observed-probably as a result of a "pulling effect" which led to substrate saturation of SsfN. MAACoA was never detected in any of the strains, even not in early phases of yeast growth, as confirmed by acyl-CoA analysis at 4 and $8 \mathrm{~h}$ (Additional file 1: Figure S3). MAACoA was also not detected in yeasts expressing genes from the thg cluster of $P$. rubra (Additional file 1: Figure S6), albeit the yeasts were able to produce AN-CoA. The activities of the enzymes ThgI, ThgK and ThgH, homologues to SsfN, SsfK and SsfJ, were characterized in vitro by Inahashi et al. [14], confirming that the AN-CoA pathway starts via MM-CoA and Ac-CoA condensation and runs through the intermediates MAA-CoA and HMBCoA. Rapid conversion of MAA-CoA to either HMBCoA or Pr-CoA and Ac-CoA (see before) might explain its analytical absence.
We have demonstrated production of AN-CoA up to $6.4 \mathrm{mg} / \mathrm{L}$ in yeast by introducing the bacterial $s s f$ pathway. However, avenues remain to explore for further optimization of S. cerevisiae-based production of ANCoA. Titres may e.g. be improved by insertion of higher copy numbers of rate limiting pathway enzymes, by promoter-based optimization of expression levels of individual enzymes, or by avoiding accumulation of pathway intermediates. Deletion of the non-essential gene pot 1 could also increase production levels. Compartmentalization of the heterologous pathway may also be a valuable approach for efficient production as it may increase spatial proximity of enzymes involved. To avoid feeding of propionic acid, insertion of a de novo production route to Pr-CoA is desirable. During submission of this manuscript, Krink-Koutsoubelis and colleagues reported a direct Pr-CoA production route from malonyl-CoA using parts of the 3-hydroxypropionate carbon assimilation cycle found in certain auxotrophic archaea and bacteria [31]. Coupling of this Pr-CoA biosynthesis pathway to the ssf pathway could enable AN-CoA production without addition of media supplements.

Saccharomyces cerevisiae has already been used for the biosynthesis of important precursors of esters of angelic acid, such as precursors of the diterpenoid ingenol-3-angelate [32]. Together with the reported AN-CoA synthesis, $S$. cerevisiae may provide an important and economic route to total biosynthesis of ingenol-3-angelate and other valuable angelates.

\section{Expression of acyl-CoA synthases from plant origin in S. cerevisiae}

We also report the identification of plant acyl-CoA synthases able to utilize angelic acid in order to yield $\mathrm{AN}-\mathrm{CoA}$. In plants AN-CoA production most probably follows a different pathway than the one found in actinomycetes. AN-CoA may be derived from degradation of L-isoleucine (via 3-methyl-2-oxopentanoate and 2-methylbutanoyl-CoA) or from tiglyl-CoA through a cis-trans isomerase system, similar to that responsible for crotonyl-CoA isomerization [33, 34]. However, none of the responsible enzymes for its biosynthesis have been identified.

Here we attempted to enable ligation of angelic acid and CoA by employing heterologous enzymes known to have CoA ligation activity and substrate promiscuity. The enzyme HICCL4 from $H$. lupulus, involved in bitter acids biosynthesis pathway in hop, was shown to have substrate preference towards several short-chain fatty acids, including isobutyric acid and 2-methylbutyric acid [35]. Obviously, the ability of HICCL4 to utilize angelic acid as substrate can be attributed to the structural resemblance of the saturated acids that are the usual preferred 
substrates of the enzyme. The enzyme StCCL, showing $71 \%$ identity to HICCL4 (Additional file 1: Figure S4), showed the highest activity and led to the highest titres of AN-CoA. Two of the enzymes identified in the transcriptome of Euphorbia peplus (EpCCL1 and EpCCL2) proved to be quite efficient in AN-CoA production, but not as much as StCCL. The Euphorbia CoA ligases are probably tightly linked to ingenol biosynthesis in the plant. Therefore, activity of those enzymes is synchronized with ingenol-3-angelate biosynthesis rather than being optimized for AN-CoA production. It may be also possible that ANCoA synthesis does not go via the intermediate angelic acid in Euphorbia peplus.

Although angelic acid feeding might not be a relevant strategy for biotechnological production of AN-CoA, we envision that the strains expressing the CoA ligases identified in this work can provide a system for screening and functional characterization of acyl-transferases able to use angelyl-CoA as donor acyl-CoA. Such enzymes are needed for the transfer of the angelate moiety onto diverse acceptor molecules. More work will be necessary to identify these enzymes. We are currently screening the transcriptome of Euphorbia peplus for identification of possible candidates involved in ingenol-3-angelate biosynthesis. Candidates for this reaction are enzymes of the BAHD family of acyltransferases, as it has been recently shown for the esterification of hydroxycinnamoyl- and benzoyl-CoA [36]. Rapid acylation by BAHDs would probably also prevent the observed disappearance of angelyl-CoA, which may be due to intracellular hydrolysis of the activated compound, as previously observed for several CoA-activated molecules [37, 38].

\section{Conclusions}

In this proof of concept study we have successfully achieved AN-CoA production in yeast by the expression of genes from the bacterial ssf cluster. This represents the first report on the activity of these enzymes in vivo. Moreover, we have identified acyl-CoA ligases from different plant species that use angelic acid as substrate and yield considerable titres of AN-CoA. Our results pave the way for future microbial production of different kinds of angelates.

\section{Methods}

\section{Chemicals and media}

All chemicals were bought from Sigma-Aldrich (St. Louis Missouri, USA) unless stated otherwise. Authentic standard of angelyl-CoA was synthesized by Jubilant LifeSciences, India.

LB medium for growth of Escherichia coli was supplied from Carl Roth GmbH + Co. KG (Karlsruhe, Germany), and was supplemented with $100 \mu \mathrm{g} / \mathrm{L}$ of ampicillin for amplification of plasmids.

Yeast extract peptone dextrose (YPD) medium with $20 \mathrm{~g} / \mathrm{L}$ glucose was used for growth of wildtype strains prior to transformation. For pre- and main cultures of transformed strains we used synthetic complete (SC) drop-out medium (Formedium LTD, Hustanton, England), supplemented with $6.7 \mathrm{~g} / \mathrm{L}$ yeast nitrogen base, $20 \mathrm{~g} / \mathrm{L}$ glucose and all amino acids necessary for the corresponding auxotrophy. For propionyl-CoA carboxylase-expressing strains medium was supplemented with additional biotin $(20 \mu \mathrm{g} / \mathrm{L})$. Basic amounts of biotin are routinely added to baker's yeast cultures as the co-factor is not produced by the laboratory strain S288C [39].

For preparation of $\mathrm{pH}$ 4.5-buffered medium a $1.0 \mathrm{M}$ stock solution of citrate buffer (sodium citrate and citric acid) was prepared. Buffer stocks were filter-sterilized and used at the final concentration of $100 \mathrm{mM}$.

Organic acid supplemented media were prepared as solutions containing $0.5 \mathrm{~g} / \mathrm{L}(6.7 \mathrm{mM})$ propionic acid, $0.5 \mathrm{~g} / \mathrm{L}(4.23 \mathrm{mM})$ methylmalonic acid, or $0.1 \mathrm{~g} / \mathrm{L}$ $(1.0 \mathrm{mM})$ angelic acid, respectively. Stock solutions $(1.0 \mathrm{M})$ of methyl-malonic acid and angelic acid were prepared in deionized water and ethanol respectively.

\section{Plasmids and strains}

Table 1 lists all plasmids constructed in this work. All coding sequences were synthesized by GeneArt ${ }^{\circledR}$ (Thermofisher Scientific, Zug, Switzerland) as yeast-codon optimized versions. Standard cloning was done using the restriction enzymes HindIII HF and SacII, and T4 DNA ligase from New England Biolabs (Ipswich, Massachusetts, USA) according to standard protocols [40]. E. coli XL10 Gold (Agilent, Santa Clara, California, USA) cells were used for subcloning of genes. Coding sequences were cloned in single expression vectors (ARS/CEN), or in entry vectors for assembly of multigene expression plasmids in vivo by homologous recombination (HRTs), as described by Eichenberger et al. [41]. Briefly, genes were cloned into entry vectors, carrying different combinations of promoters and terminators ("expression cassettes"), flanked by 60 base pair homology sequences. Helper cassettes containing (a) the autonomously replicating sequence, (b) a centromere region, and (c) the auxotrophy marker are also flanked by 60 base pair homology sequences. Expression cassettes as well as helper cassettes were released by one-pot digestions using AscI (New England Biolabs). The digested mixtures were transformed into yeast. In case of negative expression control, corresponding empty entry vectors were added to the digestion mix.

Saccharomyces cerevisiae strains generated throughout this study are listed in Table 2. All constructed strains 
Table 1 List of genes used in this work and plasmids they were cloned into

\begin{tabular}{lllll}
\hline Plasmid & Promoter and gene & Plasmid type & Organism & Accession number \\
\hline pANG1 & pPGK1-ssfN & Entry vector & Streptomyces sp. SF2575 & ADE34503.1 \\
pANG2 & pTEF1-ssfK & Entry vector & Streptomyces sp. SF2575 & ADE34504.1 \\
pANG3 & pPDC1-ssfJ & Entry vector & Streptomyces sp. SF2575 & ADE34505.1 \\
pANG4 & pGPD1-ssfE & Entry vector & Streptomyces sp. SF2575 & ADE34513.1 \\
pANG5 & pTEF1-thgK & Entry vector & Polymorphospora rubra K07-0510 & BAU79605.1 \\
pANG6 & pPDC1-thgH & Entry vector & Polymorphospora rubra K07-0510 & BAU79602.1 \\
pANG7 & pTEF2-thgl & Entry vector & Polymorphospora rubra K07-0510 & BAU79603.1 \\
pANG8 & pGPD1-pcCB & Entry vector & Streptomyces coelicolor A3(2) & NP_629079.1 \\
pANG9 & pPGK1-accA1 & Entry vector & Streptomyces coelicolor A3(2) & NP_733754.1 \\
pANG10 & pPGK1-matB & ARS/CEN & Streptomyces coelicolor & CAB86109.1 \\
pANG11 & pPGK1-prpE & ARS/CEN & Salmonella enterica serovarTyphimurium & AAC44817.2 \\
pANG12 & pGPD1-ssfE & ARS/CEN & Streptomyces sp. SF2575 & ADE34513.1 \\
pANG13 & pCYC1-prpE & ARS/CEN & Salmonella enterica serovarTyphimurium & AAC44817.2 \\
pANG14 & pTEF1-HICCL4 & ARS/CEN & Humulus lupulus & AGA17921.1 \\
pANG15 & pTEF1-StCCL & ARS/CEN & Solanum tuberosum & XP_006350454.1 \\
pANG16 & pTEF1-EpCCL1 & ARS/CEN & Euphorbia peplus & - \\
pANG17 & pTEF1-EpCCL2 & ARS/CEN & Euphorbia peplus & - \\
pANG18 & pTEF1-EpCCL3 & ARS/CEN & Euphorbia peplus & - \\
\hline
\end{tabular}

were derived from strain NCYC 3608 (NCYC, Norwich, United Kingdom), a derivative of S288C, modified in our labs to add auxotrophophic markers (HIS, LEU, URA), and repair the petite phenotype according to Dimitrov et al. [42]. All yeast strains were stored in $25 \%$ glycerol at $-80{ }^{\circ} \mathrm{C}$.

Table 2 List of strains constructed in this work

\begin{tabular}{ll}
\hline Strains & Description \\
\hline ANG1 & Control strain with empty plasmid (URA3) \\
ANG2 & Contains expression cassettes of ssfE/ssfN/ssfK/ssfJ (URA3) \\
ANG3 & Contains expression cassettes of ssfE/ssfN/ssfK/ssfJ (URA3) and an empty plasmid (LEU2) \\
ANG4 & Contains expression cassettes of ssfE/ssfN/ssfK/ssfJ (URA3) and prpE (LEU2) \\
ANG5 & Contains expression cassettes of pccB/accA1/ssfN/ssfK/ssfJ (URA3) and prpE (LEU2) \\
ANG6 & Contains expression cassettes of ssfE (URA3) and prpE (LEU2) \\
ANG7 & Contains expression cassettes of ssfE/ssfN (URA3) and prpE (LEU2) \\
ANG8 & Contains expression cassettes of ssfE/ssfN/ssfKand prpE (LEU2) \\
ANG9 & Contains expression cassettes of prpE (LEU2) and an empty plasmid (URA3) \\
ANG10 & Contains expression cassettes of matB (LEU2) and an empty plasmid (URA3) \\
ANG11 & Contains expression cassettes of ssfN/ssfK/ssfJ (URA3) and an empty plasmid (LEU2) \\
ANG12 & Contains expression cassettes of ssfN/ssfK/ssfJ (URA3) and matB (LEU2) \\
ANG13 & Contains expression cassettes of ssfE/thgK/thgH/thgl (URA3) and prpE (LEU2) \\
ANG14 & Contains expression cassette of CoA ligase from Humulus lupulus (LEU2) \\
ANG15 & Contains expression cassette of CoA ligase from Solanum tuberosum (LEU2) \\
ANG16 & Contains expression cassette of CoA ligase-1 from Euphorbia peplus (LEU2) \\
ANG17 & Contains expression cassette of CoA ligase-2 from Euphorbia peplus (LEU2) \\
ANG18 & Contains expression cassette of CoA ligase-3 from Euphorbia peplus (LEU2) \\
ANG19 & Control strain with empty plasmid (LEU2) \\
ANG20 & Contains expression cassettes of ssfE/ssfN/ssfK/ssfJ (URA3), prpE (LEU2) and an empty plasmid (HIS3) \\
ANG21 & Contains expression cassettes of ssfE/ssfN/ssfK/ssfJ (URA3), prpE (LEU2) and an additional copy of ssfE (HIS3) \\
\hline &
\end{tabular}




\section{Yeast transformation and growth}

Yeast transformation was performed using the lithium acetate method [43]. Transformants were grown on agar plates prepared with selective SC drop-out medium. Precultures were grown for $24 \mathrm{~h}$ at $30^{\circ} \mathrm{C}$ on an orbital shaker $(160 \mathrm{rpm})$. Optical density at $600 \mathrm{~nm}\left(\mathrm{OD}_{600}\right)$ of a 1:40 dilution was measured in an Ultrospec 10 table top spectrophotometer (GE Healthcare, Little Chalfont, United Kingdom). Main cultures for production of angelyl-CoA were inoculated in $25 \mathrm{~mL}$ of medium at a starting $\mathrm{OD}_{600}$ of 0.1 , and grown at $30{ }^{\circ} \mathrm{C}(160 \mathrm{rpm})$ for $12-96 \mathrm{~h}$. Media supplemented with organic acids were used exclusively for the growth of main cultures.

\section{Sample preparation}

We harvested 100 OD-units $\left(\sim 1 \times 10^{9}\right.$ cells $)$ by centrifugation at $4000 \mathrm{rpm}$ for $5 \mathrm{~min}$. Cell pellets were re-suspended in $1 \mathrm{~mL}$ of water and re-pelleted in $2 \mathrm{~mL}$ screw cap tubes. Extraction from pellets was performed as described previously [44]. Briefly, cell pellets were re-suspended in $500 \mu \mathrm{L}$ of $75 \%$ ethanol and shaken (1500 rpm) for $3 \mathrm{~min}$ at $95{ }^{\circ} \mathrm{C}$ in a Thermo-Shaker TS-100 (Axonlab, Reichenbach an der Fils, Germany). Cell debris was removed by centrifugation ( $4000 \mathrm{rpm}, 5 \mathrm{~min}$ ) and the liquid phase was transferred to a 96 deepwell microplate. The ethanol extracts were evaporated for $5 \mathrm{~h}$ at $35^{\circ} \mathrm{C}$ using a Genevac HT4 (SP Industries 935 Mearns RoadWarminster, PA18974). Dried pellets were re-solubilized in $100 \mu \mathrm{L}$ of $50 \mathrm{mM}$ ammonium acetate. Remaining debris was removed by centrifugation ( $5 \mathrm{~min}$ at $4000 \mathrm{~g}$ ), and supernatants were used for analyses.

\section{Acyl-CoAs analysis}

Analytical LC-MS was carried out using a Waters Xevo G2 XS TOF mass detector (Milford, Massachusetts, USA). Separation of the compounds was achieved on a Waters Acquity UPLC ${ }^{\circledR}$ HSS T3 C18 column $(1.7 \mu \mathrm{m}$, $2.1 \mathrm{~mm} \times 50 \mathrm{~mm}) \mathrm{kept}$ at $50{ }^{\circ} \mathrm{C}$. Mobile phases were composed of (A) 1\% acetonitrile, $99 \%$ water, $5 \mathrm{mM}$ ammonium acetate, and (B) $10 \%$ acetonitrile, $90 \%$ isopropanol, $5 \mathrm{mM}$ ammonium acetate. An elution gradient from $99 \%$ A to $0 \%$ A within 2 min at a flow rate of $0.5 \mathrm{~mL} / \mathrm{min}$ was used. The mass analyzer was equipped with an electrospray source and operated in negative mode. Capillary voltage was $1.0 \mathrm{kV}$; the source was kept at $150{ }^{\circ} \mathrm{C}$ and the desolvation temperature was $500{ }^{\circ} \mathrm{C}$. Desolvation and cone gas flow were 1000 and $150 \mathrm{~L} / \mathrm{h}$, respectively. For each compound of interest we calculated peak areas on the extracted ion chromatograms of the respective $[\mathrm{M}-\mathrm{H}]^{-}$ions, using a mass window of $0.02 \mathrm{Da}$. Angelyl-CoA, acetyl-CoA and methyl-malonyl-CoA were quantified using a linear calibration curve with authentic standards ranging from 0.03125 to $4 \mathrm{mg} / \mathrm{L}$ for all compounds. For other compounds without standards, Area-under-the-curve (AUC) values were calculated for a relative quantity of the compound. Concentration and AUC values were normalized per 100 OD units.

\section{Additional file}

Additional file 1. Additional figures and table.

\begin{abstract}
Abbreviations
KAS III: beta-ketoacyl-(acyl-carrier-protein) synthase III; ACP: acyl-carrierprotein; AN-CoA: angelyl-CoA; Ac-CoA: acetyl-CoA; MM-CoA: methylmalonyl-CoA; MAA-CoA: methyl-acetoacetyl-CoA; HMB-CoA: 3-hydroxyl2-methyl-butyryl-CoA; Pr-CoA: propionyl-CoA; CCL: carboxyl CoA ligase; HICCL4: carboxyl CoA ligase 4 from Humulus lupulus; StCCL: acyl-activating enzyme 6 from Solanum tuberosum; EpCCL1: CoA-ligase 1 from Euphorbia peplus; EpCCL2: CoA-ligase 2 from Euphorbia peplus; EpCCL3: CoA-ligase 3 from Euphorbia peplus; BAHD: BEAT (benzoyl alcohol O-acetyltransferase), AHCT (anthocyanin O-hydroxycinnamoyl transferase), HCBT (anthranilate $\mathrm{N}$-hydroxycinnamoyl/benzoyl transferase), and DAT (deacetyl vindoline 4-O-acetyltransferase).
\end{abstract}

\section{Authors' contributions}

RC carried out strain constructions, experiments, extractions, and drafted the manuscript. DF established the analysis methods and analysed all samples. NW performed initial studies for the synthesis of AN-CoA and constructed some of the strains and plasmids. RC, HH and NW designed and coordinated the study. $\mathrm{HH}$ and NW reviewed and edited the manuscript. All authors read and approved the final manuscript.

\section{Author details \\ ${ }^{1}$ Evolva SA, Duggingerstrasse 23, 4153 Reinach, Switzerland. ${ }^{2}$ Department of Plant and Environmental Sciences, University of Copenhagen, 1871 Fred-} eriksberg C, Denmark.

\section{Acknowledgements}

We thank Samantha Capewell for proofreading of the manuscript, Andreas Dallügge and Curt Nielsen for providing the genes encoding HICCL4 and StCCL, Philipp Berninger for support in bioinformatics and Professor Birger Lindberg Møller for support.

\section{Competing interests}

Roberta Callari, Harald Heider and Nora Weber declare financial competing interests in the form of a pending patent application filed by Evolva.

\section{Availability of data and materials}

The datasets used and/or analyzed during the current study are available from the corresponding author on reasonable request.

\section{Consent for publication}

Not applicable.

\section{Ethics approval and consent to participate}

Not applicable.

\section{Funding}

This work was supported by "Plant Power: Light-driven synthesis of complex terpenoids using cytochrome P450s" (12-131834) funded by the Danish Innovation Foundation (project lead, Dr. Poul Erik Jensen, University of Copenhagen, Department of Plant and Environmental Sciences, Plant Biochemistry Laboratory). 


\section{Publisher's Note}

Springer Nature remains neutral with regard to jurisdictional claims in published maps and institutional affiliations.

Received: 11 January 2018 Accepted: 7 May 2018

Published online: 12 May 2018

\section{References}

1. Weedon D, Chick J. Home treatment of basal cell carcinoma. Med J Aust. 1976;1:928.

2. Aydın AA, Zerbes V, Parlar H, Letzel T. The medical plant butterbur (Petasites): analytical and physiological (re)view. J Pharm Biomed Anal. 2013:75:220-9.

3. Fu H-Z, Li C-J, Yang J-Z, Shen Z-F, Zhang D-M. Potential anti-inflammatory constituents of the stems of Gordonia chrysandra. J Nat Prod. 2011;74:1066-72.

4. Zhang J, Li L, Jiang C, Xing C, Kim S-H, Lü J. Anti-cancer and other bioactivities of Korean Angelica gigas Nakai (AGN) and its major pyranocoumarin compounds. Anticancer Agents Med Chem. 2012;12:1239-54.

5. Andersen TB, López CQ, ManczakT, Martinez K, Simonsen HT. Thapsigargin-from Thapsia L. to mipsagargin. Molecules. 2015;20:6113-27.

6. Pickens LB, Kim W, Wang P, Zhou H, Watanabe K, Gomi S, et al. Biochemical analysis of the biosynthetic pathway of an anticancer tetracycline SF2575. J Am Chem Soc. 2009:131:17677-89.

7. Hatsu M, Sasaki T, Watabe H, Miyadoh S, Nagasawa M, Shomura T, et al. A new tetracycline antibiotic with antitumor activity. I. Taxonomy and fermentation of the producing strain, isolation and characterization of SF2575. J Antibiot. 1992:45:320-4.

8. Nakashima T, Okuyama R, Kamiya Y, Matsumoto A, Iwatsuki M, Inahashi Y, et al. Trehangelins A, B and C, novel photo-oxidative hemolysis inhibitors produced by an endophytic actinomycete, Polymorphospora rubra K070510. J Antibiot. 2013;66:311-7.

9. Ball M, Andrews SP, Wierschem F, Cleator E, Smith MD, Ley SV. Total synthesis of thapsigargin, a potent SERCA pump inhibitor. Toyoshima, C.; Nomura, H. Nature. 2002;418:605.

10. Liang X, Grue-Sørensen G, Petersen AK, Högberg T. Semisynthesis of ingenol 3-angelate (PEP005): efficient stereoconservative angeloylation of alcohols. Synlett@ Georg Thieme Verlag. 2012;23:2647-52.

11. Harel D, Khalid SA, Kaiser M, Brun R, Wünsch B, Schmidt TJ. Encecalo angelate, an unstable chromene from Ageratum conyzoides L.: total synthesis and investigation of its antiprotozoal activity. J Ethnopharmacol. 2011;137:620-5.

12. Jørgensen L, McKerrall SJ, Kuttruff CA, Ungeheuer F, Felding J, Baran PS. 14-Step synthesis of (+)-ingenol from (+)-3-carene. Science. 2013;341:878-82.

13. Hohmann J, Evanics F, Berta L, Bartók T. Diterpenoids from Euphorbia peplus. Planta Med. 2000;66:291-4

14. Inahashi Y, Shiraishi T, Palm K, Takahashi Y, Ōmura S, Kuzuyama T, et al. Biosynthesis of trehangelin in Polymorphospora rubra K07-0510: identification of metabolic pathway to angelyl-CoA. ChemBioChem. 2016;17:1442-7.

15. Luttik MA, Kötter P, Salomons FA, van der Klei IJ, van Dijken JP, Pronk JT. The Saccharomyces cerevisiae ICL2 gene encodes a mitochondria 2-methylisocitrate lyase involved in propionyl-coenzyme A metabolism. J Bacteriol. 2000;182:7007-13.

16. Pronk JT, van Linden-Beuman A, Verduyn C, Alexander Scheffers TW, van Dijken JP. Propionate metabolism in Saccharomyces cerevisiae implications for the metabolon hypothesis. Microbiology. 1994;140(Pt 4):717-22.

17. van den Berg MA, de Jong-Gubbels P, Kortland CJ, van Dijken JP, Pronk JT, Steensma HY. The two acetyl-coenzyme A synthetases of Saccharomyces cerevisiae differ with respect to kinetic properties and transcriptional regulation. J Biol Chem. 1996;271:28953-9.

18. Horswill AR, Escalante-Semerena JC. The prpE gene of Salmonella typhimurium LT2 encodes propionyl-CoA synthetase. Microbiology. 1999;145(Pt 6):1381-8.

19. Mutka SC, Bondi SM, Carney JR, Da Silva NA, Kealey JT. Metabolic pathway engineering for complex polyketide biosynthesis in Saccharomyces cerevisiae. FEMS Yeast Res. 2006:6:40-7.
20. Verduyn C, Postma E, Scheffers WA, van Dijken JP. Energetics of Saccharomyces cerevisiae in anaerobic glucose-limited chemostat cultures. J Gen Microbiol. 1990;136:405-12.

21. Mira NP, Teixeira MC, Sá-Correia I. Adaptive response and tolerance to weak acids in Saccharomyces cerevisiae: a genome-wide view. OMICS. 2010;14:525-40.

22. Rodriguez E, Gramajo H. Genetic and biochemical characterization of the $\mathrm{a}$ and $\mathrm{b}$ components of a propionyl-CoA carboxylase complex of Streptomyces coelicolor A3(2). Microbiology. 1999;145:3109-19.

23. An JH, Kim YS. A gene cluster encoding malonyl-CoA decarboxylase (MatA), malonyl-CoA synthetase (MatB) and a putative dicarboxylate carrier protein (MatC) in Rhizobium trifolii-cloning, sequencing, and expression of the enzymes in Escherichia coli. Eur J Biochem. 1998;257:395-402.

24. Pohl NL, Hans M, Lee HY, Kim YS, Cane DE, Khosla C. Remarkably broad substrate tolerance of malonyl-CoA synthetase, an enzyme capable of intracellular synthesis of polyketide precursors. J Am Chem Soc. 2001;123:5822-3.

25. Koryakina I, McArthur J, Randall S, Draelos MM, Musiol EM, Muddiman DC, et al. Poly specific trans-acyltransferase machinery revealed via engineered Acyl-CoA synthetases. ACS Chem Biol Am Chem Soc. 2013;8:200-8

26. Go MK, Chow JY, Cheung WWN, Lim YP, Yew WS. Establishing a toolkit for precursor-directed polyketide biosynthesis: exploring substrate promiscuities of acid-CoA ligases. Biochem Am Chem Soc. 2012;51:4568-79.

27. Hiltunen JK, Mursula AM, Rottensteiner H, Wierenga RK, Kastaniotis AJ, Gurvitz A. The biochemistry of peroxisomal beta-oxidation in the yeast Saccharomyces cerevisiae. FEMS Microbiol Rev. 2003;27:35-64.

28. Brock M, Buckel W. On the mechanism of action of the antifungal agent propionate. Eur J Biochem Blackwell Sci Ltd. 2004;271:3227-41.

29. Maruyama K, Kitamura H. Mechanisms of growth inhibition by propionate and restoration of the growth by sodium bicarbonate or acetate in Rhodopseudomonas sphaeroides S. J Biochem. 1985;98:819-24.

30. Man WJ, Li Y, O'Connor CD, Wilton DC. The binding of propionyl-CoA and carboxymethyl-CoA to Escherichia coli citrate synthase. Biochim Biophys Acta. 1995:1250:69-75.

31. Krink-Koutsoubelis N, Loechner AC, Lechner A, Link H, Denby CM, Voegeli $B$, et al. Engineered production of short chain acyl-coenzyme A esters in S. cerevisiae. ACS Synth Biol. 2018;7:1105-15.

32. Luo D, Callari R, Hamberger B, Wubshet SG, Nielsen MT, AndersenRanberg J, et al. Oxidation and cyclization of casbene in the biosynthesis of Euphorbia factors from mature seeds of Euphorbia lathyris L. Proc Natl Acad Sci USA. 2016:113:E5082-9.

33. Crout DHG. Pyrrolizidine alkaloids. Biosynthesis of the angelate component of heliosupine. J Chem Soc R Soc Chem. 1967;0:1233-4.

34. Mcgaw BA, Woolley JG. The biosynthesis of angelic acid in Cynoglossum officinale. Phytochemistry. 1979;18:1647-9.

35. Xu H, Zhang F, Liu B, Huhman DV, Sumner LW, Dixon RA, et al. Characterization of the formation of branched short-chain fatty acid:CoAs for bitter acid biosynthesis in hop glandular trichomes. Mol Plant. 2013:6:1301-17.

36. Eudes A, Mouille M, Robinson DS, Benites VT, Wang G, Roux L, et al. Exploiting members of the BAHD acyltransferase family to synthesize multiple hydroxycinnamate and benzoate conjugates in yeast. Microb Cell Fact. 2016:15:198.

37. Nomura G, Yoshida Y, Takamura Y. Measurement of the intracellular concentration of total acyl-CoA extracted by different procedures from Gram-negative and Gram-positive bacteria, and yeasts. Agric Biol Chem. 1988;52:843-4

38. Takamura Y, Nomura G. Changes in the intracellular concentration of acetyl-CoA and malonyl-CoA in relation to the carbon and energy metabolism of Escherichia coli K12. J Gen Microbiol. 1988;134:2249-53.

39. Bracher JM, de Hulster E, Koster CC, van Broek M, Daran J-MG, van Maris AJA, et al. Laboratory evolution of a biotin-requiring Saccharomyces cerevisiae strain for full biotin prototrophy and identification of causal mutations. Appl Environ Microbiol. 2017:83:e00892-17.

40. Green MR, Sambrook J. Molecular cloning: a laboratory manual, 4th edn, vol. 1 Ch. 9. Cold Spring Harbour Laboratory Press; 2012. p. 157-260.

41. Eichenberger M, Lehka BJ, Folly C, Fischer D, Martens S, Simón E, et al. Metabolic engineering of Saccharomyces cerevisiae for de novo production of dihydrochalcones with known antioxidant, antidiabetic, and sweet tasting properties. Metab Eng. 2017;39:80-9. 
42. Dimitrov LN, Brem RB, Kruglyak L, Gottschling DE. Polymorphisms in multiple genes contribute to the spontaneous mitochondrial genome instability of Saccharomyces cerevisiae S288C strains. Genetics. 2009;183:365-83.

43. Gietz RD, Schiestl RH. High-efficiency yeast transformation using the LiAc/SS carrier DNA/PEG method. Nat Protoc. 2007;2:31-4.
44. Seifar RM, Ras C, Deshmukh AT, Bekers KM, Suarez-Mendez CA, da Cruz $A L B$, et al. Quantitative analysis of intracellular coenzymes in Saccharomyces cerevisiae using ion pair reversed phase ultra high performance liquid chromatography tandem mass spectrometry. J Chromatogr A. 2013;1311:115-20.
Ready to submit your research? Choose BMC and benefit from:

- fast, convenient online submission

- thorough peer review by experienced researchers in your field

- rapid publication on acceptance

- support for research data, including large and complex data types

- gold Open Access which fosters wider collaboration and increased citations

- maximum visibility for your research: over $100 \mathrm{M}$ website views per year

At BMC, research is always in progress.

Learn more biomedcentral.com/submissions 